\title{
Aproximación desde la psicología y la educación a las actividades de programación y robótica educativa
}

\section{An approach from psychology and education to programming and educational robotics activities}

\author{
Esther Angeriz ${ }^{1}$
}

Fecha de recepción: 4 de abril de 2020

Fecha de aceptación: 9 de octubre de 2020

\section{Resumen}

Este trabajo plantea una reflexión crítica sobre las relaciones entre la psicología, la educación y los recursos tecnológicos, en especial referidos a las actividades de robótica educativa y programación. Se enmarca en una consideración general que identifica a la educación como uno de los campos que se encuentra tensionado en función de una realidad social acuciante, con consecuencias políticas, económicas y subjetivas, en la cual las TIC se han convertido, circunstancialmente, en protagonistas casi exclusivas, dada su posibilidad de mediar y tender puentes en los vínculos humanos. Se presentan perspectivas desde la psicología y la educación en relación con la alfabetización necesaria en el siglo $\mathrm{XXI}$, debates en torno a constructos emergentes, como el pensamiento computacional, y con las posibilidades de la robótica educativa y la programación en el marco de las competencias del siglo XXI y de los procesos de apropiación de la tecnología. En las consideraciones finales, se fundamenta la inclusión de la robótica en la educación como una de las posibles formas de construir conocimiento en la actualidad, pero a través de una formación que trascienda objetivos exclusivamente tecnológicos que apunte a una formación integral y sensibilice a los estudiantes con los problemas de su comunidad, en un marco de igualdad de

\footnotetext{
${ }^{1}$ Magíster en Psicología y Educación. Doctoranda del Programa de Doctorado en Psicología de la Facultad de Psicología. UdelaR. Profesora Adjunta del Instituto de Psicología, Educación y Desarrollo Humano. Facultad de Psicología. UdelaR. Correo electrónico: eangeriz@psico.edu.uy
} 
oportunidades y de educación democratizadora en materia no solo de acceso a recursos tecnológicos, sino de construcción de competencias necesarias en la actualidad.

Palabras clave: psicología, educación, programación y robótica educativa.

\section{Abstract}

This work addresses a critical reflection on the relationships between psychology, education and technological resources, especially those related to educational robotics and programming activities. It is framed in the context of education, considering it is one of the fields that is oppressed by reality, with political, economic and subjective consequences, in which ICTS have become protagonists given their possibility of mediating and building bridges between people. Perspectives about education and digital literacy, discussions about emerging concepts such as computational thinking, related to educational robotics and programming possibilities within the framework of the $21^{\text {st }}$ century competencies, and of technology appropriation are presented. The inclusion of robotics in education is founded as one of the possible ways of constructing knowledge today, but it must be oriented towards a comprehensive education that sensitizes students regarding the issues of their community. All of this must be included within the framework of democratizing education, not only in access to technological resources, but also in the construction of currently required competences.

Keywords: psychology, education, programming and educational robotics

Introducción: la sociedad y la educación en la encrucijada de la segunda década del siglo XXI

La actual sociedad, tan interconectada como desigual, ya no puede ocultar las facetas más dramáticas de la globalización y del capitalismo salvaje, con la transnacionalización de problemas sociales relacionados con los movimientos migratorios y situaciones de extrema pobreza, con la explotación indiscriminada de hábitats naturales, así como con la salud de la población mundial puesta en jaque por enfermedades infecciosas emergentes y reemergentes que ya habían sido alertadas por la oms desde el año 2001 (Alonso, 2003). 
Llegados al punto en que el exterior, lo extraño y el vínculo con los otros son categorías de lo peligroso y amenazante, la única manera de recuperar el sentido de lo social en su condición humana es reinstalar la ética en el sentido levinasiano de asumir la responsabilidad frente al otro (Fernández Agis, 2012), reconociendo la presencia del semejante en su alteridad. Desde este enfoque, compete también a la educación reinstalar esta dimensión, teniendo en cuenta la función de la escuela de producir subjetividades, lo cual, al decir de Bleichmar (2008), no puede ser reemplazado por ninguna tecnología. Se trata, pues, de apostar a la construcción del sujeto ético en contraposición al sujeto disciplinado homogéneo en el sentido de reconstruir al semejante, de comprender las posibilidades dentro de las legalidades, de dar lugar en la actividad colectiva a la discusión de reglas, acuerdos sobre la convivencia o sobre los objetivos de integrar y usar determinados artefactos y recursos (Erausquin, 2017).

Partiendo de estos supuestos iniciales se reflexiona, en este trabajo, sobre la integración en la educación de recursos derivados de las tecnologías de la información y la comunicación (TIC) y de conceptos relativos a la alfabetización digital. Asimismo, se entiende que es uno de los campos que se tensiona en función de esta realidad social acuciante, que trae consecuencias políticas, económicas y subjetivas, en la cual las TIC se han convertido, circunstancialmente, en las protagonistas casi exclusivas, dada su posibilidad de mediar y tender puentes en los vínculos humanos.

Si bien la expansión tecnológica es casi un fenómeno universal, no todas las personas pueden aprovechar las potencialidades de las TIC; muchos son los desafíos, tensiones y brechas derivadas de este fenómeno. Los autores van Dijk y van Deursen (2014) sostienen que aun cuando en cierta medida la brecha digital se puede estar cerrando en términos de acceso material, se está profundizando por la brecha existente en materia de habilidades digitales y por la desigualdad en el uso diario de la tecnología digital. Esto da cuenta del riesgo de que el avance tecnológico y la desigual participación en torno a él refuercen y profundicen las desigualdades (Rivoir, Rivero y Pittaluga, 2011; van Dijk, 2005), en tanto la gran mayoría de la población participa en el mercado de consumo de tecnología, pero solo la minoría puede generar vínculos en términos de competencias que le permitan apropiarse de estos recursos tecnológicos (Cabello, 2017).

En estas circunstancias, la problemática de promover factores de equidad a través de la educación, fomentando la alfabetización necesaria en la actualidad, sigue siendo una preocupación política del Estado y de todos los actores que puedan aportar una mirada al problema. En este sentido, cabe preguntarse: ¿qué 
Angeriz - Aproximación desde la psicología y la educación a las actividades...

características debe tener esta alfabetización? ¿Qué es lo que necesita saber, conocer y hacer propio una persona para lograr una inserción activa, productiva, reflexiva y solidaria en la sociedad?

\section{Retos de la alfabetización en el siglo xxI}

La expresión alfabetización digital, surgida en los años noventa, integra los nuevos requerimientos y lenguajes que es preciso incorporar, puesto que incluye la capacidad de leer y comprender textos hipertextuales y multimediales (Bawden, 2002). Además de nuclear en esta definición requerimientos sobre lo que se debe aprender, es importante incluir otras dimensiones que tienen que ver con lo que, eventualmente, la alfabetización debiera posibilitar en términos de la reflexividad de un sujeto sobre sus prácticas. En ese sentido, la especialista Emilia Ferreiro (2011) sostiene que alfabetizar no es aprender el código lecto-escrito, sino que implica una reconversión conceptual a partir de la cual el lenguaje, que se aprende como medio de comunicación, se convierte en un objeto sobre el cual se puede pensar.

De esta manera, alfabetización, reflexividad, interacción y prácticas sociales son términos que deberían ir de la mano. Desde la perspectiva de la psicología sociocultural, existe una interacción y una construcción mutua entre los sistemas culturales de representación y las formas de pensar y conocer interiorizadas (Coll y Monereo, 2011), por lo cual la modificación de los contextos mediados por las TIC va a implicar transformaciones en las modalidades de pensar y conocer de los aprendices.

La mediación con las TIC tiene la potencialidad de transformar las formas de pensar y de extender las posibilidades de acción de las personas con miras a una inserción activa en la realidad, pero de aquí a que el aprovechamiento de estas oportunidades alcance equitativamente a los distintos sectores de la sociedad hay un gran trecho. De esta manera, la problemática de la alfabetización en la actualidad, en un contexto de desigualdades, se anuda con las preocupaciones tanto en torno a la apropiación de las tecnologías y las competencias necesarias a desarrollar desde la educación, como a la apuesta por el lazo social.

Tan importante como alfabetizar en los nuevos lenguajes, fomentar la apropiación de la tecnología o impulsar las denominadas competencias del siglo XXI es seguir apostando, hoy más que nunca, a la educación en su función de construcción del lazo social. Graciela Frigerio (2008) propone que educar es una acción jurídica que implica inscribir al sujeto en lo social, otorgarle una filiación simbólica; pero también es una acción política, en tanto involucra la distribución de herencias. 
Educar implica concretar políticas que tienen que ver con el acceso a la cultura, pero también con un trabajo de conocimiento y reconocimiento, de memoria, para dar lugar al otro como semejante, con sus deseos y su singular relación con los saberes (Frigerio, 2008). En este sentido, las políticas educativas deben estar orientadas desde el Estado hacia la alfabetización digital relacionando objetivos de la educación en términos de producción de subjetividades, con fines de inclusión y fortalecimiento del lazo social.

\section{El papel transformador de las TIC en las prácticas educativas y las conceptualizaciones emergentes}

Las políticas educativas relacionadas con la introducción de tecnologías digitales, que la mayoría de los países ha desarrollado desde fines de los noventa y con mayor intensidad a principios del siglo XXI, han generado transformaciones en las escuelas, en las prácticas educativas, así como en algunos casos en los currículos. Distintos autores hacen referencia a que estas transformaciones podrían fomentar el desarrollo de competencias del siglo XXI (Cabero y Llorente, 2008; Coll, 2004; Gros, 2000); sin embargo, también se plantea que la incorporación de estas tecnologías no siempre transforma efectivamente las prácticas educativas (Cobo, 2016). De acuerdo con Coll y Monereo (2011). Esto no debe rebajar las expectativas que se tienen sobre el valor transformador de las TIC en la educación, en tanto se trata de un potencial que puede o no desarrollarse en función de los contextos en los que se utiliza, los objetivos con que se plantea y lo que estudiantes y docentes hacen con ellas.

Para analizar, pues, el valor transformador de las TIC en la educación es necesario generar visibilidad en torno a los ejes que tensionan las prácticas educativas en las que se integran estas tecnologías, uno de los cuales tiene que ver con los objetivos de formación. Las tensiones entre una formación funcional para el Estado y una formación orientada a la emancipación se agudizan cada vez más cuando se trata de la integración de las TIC en la educación. Según D'Angelo (2018), el cambio en la educación en favor de una formación emancipadora depende de que se la oriente hacia una formación integral y no solo técnica, donde el interés esté puesto en las personas y no en los programas.

Otro de los ejes tiene que ver con los objetivos de aprendizaje y las perspectivas que se postulen para la integración de las TIC en la educación, que no debieran estar basados solo en lo motivante o divertida que puede resultar para los y las estudiantes la mediación con recursos que ofrecen las Tic. Según D'Angelo (2018), el entusiasmo inicial no implica que se haya adquirido conocimientos distintos a 
los que obtendrían en una clase tradicional. Para la autora, aquellos diseños pedagógicos que puedan producir efectivos aprendizajes son los que se sustenten en teorías psicológicas sobre la construcción de formas de pensar que den lugar a la transferencia de aprendizajes y a la conexión de conocimientos en distintos dominios (D'Angelo, 2018).

En ese sentido, como no existe evidencia que relacione directamente el uso de las TIC con mejores rendimientos o aprendizajes, no se debe «promover un concepto de tecnología como entidad todopoderosa determinista», según D'Angelo (2018, p.119), sino como una construcción que es dinámica, que es fruto de decisiones y que, por tanto, puede ser discutible y modificada. En definitiva, D'Angelo (2018) acepta, por un lado, la necesidad de que se desarrollen cambios en la enseñanza centrados en la actividad de los y las estudiantes en los que estén incluidos la tecnología, pero, por otro, advierte que no es ella misma la que va a producir modificaciones profundas.

En esta línea, se puede encontrar en la literatura discusiones referidas a aspectos teóricos relativos a la fundamentación psicológica y pedagógica de actividades mediadas por recursos tecnológicos, a aspectos prácticos reflejados en el día a día de las escuelas referentes a los objetivos pedagógicos del uso de estas tecnologías, a aspectos instrumentales correspondientes a la conveniencia del empleo de determinados dispositivos tecnológicos, así como a nuevos constructos emergentes de la educación con tecnologías, como el del pensamiento computacional. Muchas de las investigaciones de los últimos años sobre los temas referidos a la alfabetización digital y a las competencias del siglo xxI confluyen en este constructo, planteándose debates sobre la necesidad de introducirlo o no en los currículos escolares.

\section{Aproximaciones $y$ debates en torno al constructo del pensamiento computacional}

Sin la pretensión de realizar un análisis exhaustivo, corresponde señalar brevemente que la expresión pensamiento computacional fue introducida por Wing (2006) para referirse al proceso de pensamiento involucrado en la formulación de problemas y soluciones que puedan ser representadas y llevadas a cabo por un procesador de información. El concepto fue trabajado en un informe sobre el alcance y la naturaleza del pensamiento computacional, del Committee for the Workshops on Computational Thinking del National Research Council en Washington, D. C., donde se integraron conceptos básicos para la ciencia computacional, y se lo describió como un pensamiento que permite la resolución de problemas, el diseño de sistemas y la comprensión de los comportamientos 
humanos (como se citó en Adell Segura, Llopis Nebot, Esteve Mon y Valdeolivas Novella, 2019).

En una revisión de la literatura reciente sobre pensamiento computacional realizada por los autores Moreno-León, Román-González y Robles (2018) se señala que las definiciones del constructo se relacionan con la alfabetización computacional o digital $y$, aunque muchas de ellas han establecido como ruta el pensamiento algorítmico, el término ha ido evolucionado abarcando otros niveles de abstracciones y aplicaciones. Destacan, no obstante, que la expresión ha recibido críticas por su ambigüedad y vaguedad y que, inclusive, algunos investigadores argumentan que es una nueva forma de reunir habilidades relacionadas con la programación, que refuerza una visión estrecha del campo de la ciencia computacional (Moreno-León, Román-González y Robles, 2018).

Por su parte, Adell Segura et al (2019) se preguntan si es necesario desarrollar la habilidad de pensar computacionalmente. Sostienen los autores que la fundamentación de integrar el pensamiento computacional en la formación se alinea con dos perspectivas diferentes respecto de los fines de la educación. Por un lado, aquella que prioriza objetivos de su inclusión orientados a la adquisición de habilidades que demanda el mercado de trabajo y, por otro, la que promueve su integración para desarrollar la capacidad expresiva y comunicativa de los y las estudiantes. Sin embargo, afirman que no encuentran fundamentaciones referidas a la necesidad de desarrollar una competencia digital crítica que habilite a los y las estudiantes a construir un proyecto de vida dentro de una sociedad con profundos cambios económicos, políticos y culturales. Esto se vuelve más importante aún en el momento actual de esta sociedad que se encuentra desafiada por la posibilidad de mantener el lazo social y la sensibilidad humana a través de vínculos solidarios y empáticos con los otros, ante una situación de emergencia sanitaria de la humanidad, como lo es una pandemia.

Las actividades de robótica educativa en la construcción de competencias del siglo xxI: posibilidades y tensiones

Mientras estas discusiones académicas se procesan, en las políticas educativas de distintos países de Europa y América Latina se está planteando la necesidad de incluir en los tránsitos curriculares prácticas que integren estos nuevos lenguajes propios de la cultura tecnológica actual, dentro de las cuales se encuentran aquellas vinculadas con la robótica educativa y la programación.

Area Moreira (2017), en un análisis sobre la transformación de los materiales didácticos en el contexto actual, sostiene que el libro de texto, material didáctico hegemónico en la escuela moderna, respondía a un modelo cultural donde el 
conocimiento aparecía como empaquetado y se distribuía e insertaba de manera estandarizada en los currículos escolares. En este contexto, el autor identifica a los robots educativos y a otros recursos tecnológicos, como aquellos que permiten que los y las estudiantes no sean solo usuarios, sino diseñadores o creadores. El trabajo con robótica implica una elaboración simultánea de la programación que permita manipular el objeto tecnológico a través de órdenes, por lo cual el potencial educativo es muy grande. Se configura, según el autor, no solo una práctica didáctica relevante, sino también un campo de estudio importante, en el que entran en juego conceptualizaciones como el pensamiento computacional, los makerspaces, la programación y la robótica educativa.

A pesar de los beneficios que identifican los distintos autores citados sobre la inclusión de la robótica educativa, se plantean cuestionamientos en diferentes dimensiones; uno de ellos tiene que ver con los objetivos que se persiguen y con el hecho de que, en verdad, no se generaría una mano de obra especializada en tecnología, disponible para insertarse en el mercado y en el mundo empresarial (Acuña Zúñiga, 2009; Adell Segura et al, 2019). Según Acuña Zúñiga (2009), no resulta sostenible en el tiempo justificar la inclusión de la robótica en la educación en función de las necesidades del mercado, teniendo en cuenta que los cambios tecnológicos van a una velocidad mucho mayor de la que pueden implicar las transformaciones en los currículos escolares. Por este motivo, la inclusión de la robótica en la educación debe darse en función de las competencias que se desea crear o favorecer en los y las estudiantes (Acuña Zúñiga, 2009).

En el contexto uruguayo, muchas son las experiencias emergentes del Plan Ceibal, a través de distintos programas y docentes que han trabajado con sus grupos y han orientado a equipos de estudiantes en proyectos que lograron reconocimientos nacionales e internacionales. En esta línea, José Miguel García y Diego Castrillejo (2011) —pioneros en el trabajo y la reflexión sobre la inclusión de recursos tecnológicos y de robótica en la educación - también reivindican el uso de la robótica en el marco de un aprendizaje global y no exclusivamente tecnológico o de formación para el trabajo; apuntan a una formación que sensibilice a los estudiantes en cuanto a problemáticas generalmente locales y de su propia comunidad, abriendo posibilidades en lo que refiere a sus respuestas, tal como lo exponen:

El punto pasa por generar posibilidades, de eso se trata la educación; no se cambiará la ecuación de un día para otro, pero se logra agregar algún grado de libertad que permita a los alumnos crecer conscientes de sus límites y de los límites externos, que inciden pero no determinan sus elecciones y su desarrollo (2011, p. 317). 
En otro orden, se plantean discusiones que tienen que ver con las estrategias pedagógicas y los objetivos de formación que sustentan las actividades de programación y robótica educativa. Gran parte de los equipos de robótica suelen estar acompañados de modelos preestablecidos, con tutoriales que los y las estudiantes deben seguir para su armado y su funcionamiento. Según García y Castrillejo (2011), ello suprime las posibilidades de la imaginación al partir de un objeto prediseñado, restringiendo también los procesos cognitivos que unen las distintas etapas que, a su entender, componen el trabajo con robótica; estos son: imaginar, diseñar, construir y programar. Se trata de etapas que no son lineales, porque el pasaje de la elaboración de la idea a su concreción puede mostrar aspectos que no funcionan, lo que da lugar a ajustes del diseño original, transformando también el producto final y la solución a la que se aspira (García y Castrillejo, 2011). Desde la perspectiva de los autores, el hecho de que la estrategia pedagógica se oriente al seguimiento de determinados pasos para construir el robot tal como fue diseñado en el manual va a atentar contra el desarrollo de la creatividad, la autonomía, la incorporación de estrategias para resolver los problemas, entre otros diversos aprendizajes.

Otro aspecto que tensiona las prácticas y que alude también a los fundamentos pedagógicos de la robótica educativa tiene que ver con el lugar del y la docente en estas propuestas. Barranco Candanedo (2012) plantea que la motivación e interés de esta figura es fundamental en la obtención de los mejores resultados del trabajo de los y las estudiantes, la permanencia, el desarrollo de la creatividad, la imaginación y la autonomía en los aprendizajes. En este sentido, García y Castrillejo (2011) plantean que, además, los y las docentes no conocen cuál debe ser el producto final al que se llegue y que, mientras los estudiantes ganan en autonomía, los primeros pierden el control sobre su proyecto, lo que implica que aprenden junto con sus estudiantes. De esta manera, la función del y la docente no estará orientada a dirigir el proceso para alcanzar determinado resultado, sino a colaborar en aspectos asociados al proceso en sí, como, por ejemplo, regular las frustraciones para que no obstaculicen el proceso y, por el contrario, lo motiven. Esta modalidad implica el reconocimiento de que los y las docentes no lo conocen todo $\mathrm{y}$, en especial, de no dejarse encandilar por las tecnologías, sino concentrarse en los procesos; se trataría desde este enfoque de dar lugar a lo que Papert denominó profesor-aprendiz (como se citó en García y Castrillejo, 2011).

Quiere decir que se puede considerar como posibilidades de la integración de este tipo de actividades en la educación una complejidad de aprendizajes que tienen que ver con la creatividad, el desarrollo de la imaginación, con un aprendizaje horizontal y autónomo - cuya evaluación aún no está considerada dentro de los 
modelos tradicionales-, mientras que algunos de los ejes que tensionan estas prácticas se dirimen entre una formación exclusivamente tecnológica y una formación sensible a los problemas locales en los cuales la utilización de estas herramientas y recursos tecnológicos adquiera sentido.

\section{Consideraciones finales}

Desde distintas perspectivas se apoya la idea de que la integración de las TIC y, en especial en la educación de recursos tecnológicos programables como los de robótica educativa, tienen la potencialidad de producir cambios en las formas de pensar y de construir conocimiento de los y las estudiantes. La inclusión de estas actividades se visibiliza como una de las posibles formas de construir conocimiento en la actualidad, a partir de la construcción de objetos simulados de la realidad que permitan pasar de lo concreto a lo abstracto, asociado al aprendizaje de lenguajes propios de la cultura tecnológica: lenguajes de programación, codificación, lenguaje audiovisual, entre otros. No obstante, como plantean García y Castrillejo (2011), también es necesario que estas actividades estén orientadas hacia una formación que sensibilice a los y las estudiantes sobre los problemas de su comunidad y los posicione desde el lugar de protagonistas, con la capacidad de dar respuesta a esas problemáticas locales.

Sin embargo, las discusiones y las tensiones no están laudadas. Como plantean Adell Segura et al. (2019) es necesario generar más investigación, conocer las prácticas y las perspectivas de los actores para que los enfoques no se orienten hacia una formación exclusivamente tecnológica, sino que estén fundamentados en perspectivas integrales de formación, contextualizadas, que promuevan la comprensión de los nuevos lenguajes y la apropiación de las Tic, así como también una reflexión crítica que ponga en discusión cuál es su incidencia en la vida de las personas. Esto implica políticas educativas que brinden igualdad de oportunidades en la formación de los y las estudiantes de acuerdo con los requerimientos actuales de la alfabetización. Desde el Estado se debe proporcionar soportes infraestructurales del acto educativo en la enseñanza pública a través del apoyo necesario a los y las docentes, de los recursos para que los y las estudiantes desarrollen las competencias y los conocimientos requeridos en función de sus contextos, promoviendo la participación social y comunitaria aun en situaciones límites de distanciamiento social.

Siguiendo a Adell Segura et al. (2019) la enseñanza de habilidades relativas a la programación implica una decisión política que debe trascender las propuestas dirigidas únicamente a la capacitación técnica y a la formación de ciudadanos y ciudadanas aptos para el mercado. Debe permitir visibilizar las tensiones e 
Angeriz - Aproximación desde la psicología y la educación a las actividades...

intereses que están detrás de los algoritmos para comprender su incidencia en nuestros proyectos vitales y estos, a su vez, se explican de la siguiente manera:

Comprender qué es un algoritmo requiere también comprender por qué nuestra vida y nuestras relaciones, por ejemplo, con la administración y el poder, están cada vez más mediatizadas por sistemas algorítmicos, en qué tipo de supuestos se basan dichos sistemas, qué consecuencias tienen en nuestras vidas y qué derechos debemos reivindicar. Enseñar a programar no es una decisión neutral, científica y objetiva. Es una decisión política y las propuestas habituales, dirigidas únicamente a la capacitación operacional y técnica, refuerzan la visión neoliberal de la educación como únicamente una inversión en capital humano $(2019$, p. 15)

\section{Bibliografía}

Acuña Zúñiga, A. L. (2009). La robótica educativa: un motor para la innovación. San José, Costa Rica: Fundación Omar Dengo. Recuperado de https://docplayer.es/13152634-La-robotica-educativa-un-motor-para-la-innovaci on.html

Adell Segura, J., Llopis Nebot, M. Á., Esteve Mon, F. M. y Valdeolivas Novella, M. G. (2019). El debate sobre el pensamiento computacional en educación. RIED. Revista Iberoamericana de Educación a Distancia, 22(1), 171-186. doi: http://dx.doi.org/10.5944/ried.22.1.22303.

Alonso, D. (2003). Globalización y enfermedades infecciosas. Real Instituto Elcano. Estudios internacionales y estratégicos. Recuperado de http://www.realinstitutoelcano.org/wps/portal/rielcano_es/contenido/!ut/p/a1/04_ Sj9CPykssy0xPLMnMz0vMAfGjzOKNQ1zcA73dDQ38_YKNDRwtfN1cnf2cDf1Dj fULshOVAepxmvs!/?WCM_GLOBAL_CONTEXT=/elcano/Elcano_es/Zonas_es/ 0012

Area Moreira, M. (2017). La metamorfosis digital del material didáctico tras el paréntesis Gutenberg. RELATEC: Revista Latinoamericana de Tecnología Educativa, 16(2), 13-28. doi: https://doi.org/10.17398/1695-288X.16.2.13

Barranco Candanedo, A. A. (2012). La robótica educativa, un nuevo reto para la educación panameña. Teoría de la Educación. Educación y Cultura en la Sociedad de la Información, 13(2), 9-17. Recuperado de https://www.redalyc.org/pdf/2010/201024390002.pdf

Bawden, D. (2002). Revisión de los conceptos de Alfabetización Informacional y alfabetización digital. Anales de Documentación, 5(0), 361-408. Recuperado de http://revistas.um.es/analesdoc/article/view/2261

Bleichmar, S. (2008). Violencia social, violencia escolar: de la puesta de límite a la construcción de legalidades. 1a. ed. Buenos Aires, Argentina: Centro de Publicaciones Educativas y Material Didáctico. 
Angeriz - Aproximación desde la psicología y la educación a las actividades...

Cabello, R. (2017). Introducción. La comprensión de los vínculos que establecemos con las tecnologías. En R. Cabello y A. López. Contribuciones al estudio de procesos de apropiación de tecnologías (pp. 11-24). Buenos Aires, Argentina: Rada Tilly: Del Gato Gris, Red de Investigadores sobre Apropiación de Tecnologías. Recuperado de http://www.delgatogris.com.ar/wp-content/uploads/2017/10/Cabello-y-L\%C3\%B 3pez-eds-Contribuciones-al-estudio-de-procesos-de-apropiaci\%C3\%B3n-de-tec nolog\%C3\%ADas.pdf

Cabero, J., Llorente, M. C. (2008). La alfabetización digital de los alumnos. Competencias digitales para el siglo XXI. Revista Portuguesa de Pedagogía. 42 (2), 7-28. Recuperado de http://hdl.handle.net/10316.2/4673

Cobo, C. (2016) La Innovación Pendiente. Reflexiones (y Provocaciones) sobre educación, tecnología y conocimiento. Montevideo, Uruguay: Colección Fundación

Ceibal/Debate. file://C:/Users/Esther/AppData/Local/Temp/La_innovacion_pendiente.pdf

Coll, C. (2004). Psicología de la educación y prácticas educativas mediadas por las tecnologías de la información y la comunicación. Una mirada constructivista. Revista Electrónica Sinéctica. 25, 1-24. Instituto Tecnológico y de Estudios Superiores de Occidente. Jalisco, México. Recuperado de https://www.redalyc.org/pdf/998/99815899016.pdf

Coll, C. y Monereo, C. (2011). Psicología de la educación virtual. Madrid, España: Ediciones Morata.

D'Angelo, V. S. (2018). La programación de ordenadores. Reflexiones sobre la necesidad de un abordaje interdisciplinar. Revista Iberoamericana de Ciencia, Tecnología y Sociedad - CTS. 13(39), 111-141. Recuperado de http://ojs.revistacts.net/index.php/CTS/article/view/83

Erausquin, C. (Julio de 2017). ¿Qué hace la diferencia en la construcción del sujeto ético? Experiencias y entramados psico-educativos. En J. J. Romero (Presidencia) Inclusión y equidad para el bienestar de las Américas. Conferencia llevada a cabo en el Congreso Interamericano de Psicología 2017. Sociedad Interamericana de Psicología (SIP), Mérida, México.

Fernández Agis, D. (2012). La ética de Lévinas, un pensamiento de la responsabilidad. Eikasia. Revista de filosofía. 45, 161-170. Recuperado de http://www.revistadefilosofia.org/45-09.pdf

Ferreiro, E. (2011). Alfabetización digital. ¿De qué estamos hablando? Educação e Pesquisa. 37(2), 425-438. doi: http://dx.doi.org/10.1590/S1517-97022011000200014

Frigerio, G. (Junio de 2008). Formar para el ejercicio de la enseñanza. Preguntas alrededor de la problemática del saber. En. F. Cross, J. Bolon (Presidencia) La professionnalisation des enseignants de l'éducation de base: les recrutements 
sans formation initiale. Conferencia llevada a cabo Seminario Internacional. Centre international d'études pédagogiques (CIEP), Sèvres, Francia.

García, J. M. y Castrillejo, D. (2011). Los robots como excusa. En Baez, J. M. García, G. Rabajoli (comp.) El modelo Ceibal. Nuevas tendencias para el aprendizaje. Montevideo, Uruguay: Centro Ceibal-ANEP.

Gros, B. (2000). El ordenador invisible. Hacia la apropiación del ordenador en la enseñanza. Barcelona, España: Gedisa.

Moreno-León, J., Román-González and M., Robles, G. (April 2018) On computational thinking as a universal skill: $A$ review of the latest research on this ability (pp. 1684-1689). En C. González, M. Castro and M. Llamas (Co-chairs). Conferencia llevada a cabo en el IEEE Global Engineering Education Conference (EDUCON).Tenerife, Spain. doi: 10.1109/EDUCON.2018.8363437

Rivoir, A., Rivero M., Pittaluga L. (2011). Informe de Investigación El Plan Ceibal: Impacto comunitario e inclusión social 2009 - 2010. Recuperado de http://www.observatic.edu.uy/inicio?p=437

van Dijk, J.A.G.M., van Deursen A.J.A.M. (2014) Defining Internet Skills. In A.J.A.M. van Deursen and J.A.G.M van Dijk Digital Skills. Palgrave Macmillan's Digital Education and Learning (pp. 21-42), New York, U.S.: Palgrave Macmillan. doi: https://doi.org/10.1057/9781137437037_2

van Dijk, J. (2005). The deepening divide. Inequality in the information society. London, UK: Thousand Oaks: Sage Publications. DOI:http://dx.doi.org/10.4135/9781452229812

Wing, J. M. (2006). Computational thinking. Communications of the ACM, 49 (3), 33-35. doi: https://doi.org/10.1145/1118178.1118215. 